ISSN: 2174-5609

DOI. http://dx.doi.org/10.14198/INTURI2016.12.06

jur Investigaciones

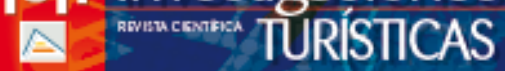

\title{
La intermodalidad aéreo-ferroviaria del turismo en la ciudad de Toledo ${ }^{1}$
}

\author{
Ana Isabel Muro Rodríguez \\ anaisabel.muro@uclm.es \\ Israel Roberto Pérez Jiménez \\ iroberto.perez@uclm.es
}

Universidad de Castilla-La Mancha, España

\section{RESUMEN}

Con la apertura de la nueva infraestructura en Madrid que comunica las estaciones de Atocha y Chamartín con el Aeropuerto de Madrid, la ciudad de Toledo se encuentra directamente conectada con el aeropuerto internacional de Madrid, lo que abre nuevas posibilidades de oferta turística con la capacidad de fomentar el turismo internacional para la ciudad de Toledo. Teniendo en cuenta la importancia de la conexión con el aeropuerto internacional para la ciudad de Toledo, unido a la nueva tendencia de creación de líneas de autobuses directos al aeropuerto desde diferentes ciudades y a las recientes ofertas de billetes combinados TAV-Avión en otros corredores, este estudio se centra en la movilidad intermodal, en concreto aéreo-ferroviaria, del turista que accede a la ciudad de Toledo. La metodología utilizada es el análisis descriptivo de los datos obtenidos a través de una encuesta realizada en el corredor, que permite el análisis del modo de transporte utilizado por los turistas, en el acceso a la ciudad de Toledo y en particular desde el aeropuerto de Madrid.

Palabras clave: Intermodalidad del turismo, movilidad del turismo, ciudad de Toledo.

\footnotetext{
${ }^{1}$ Este Trabajo se ha desarrollado en el marco del Proyecto "Integración del transporte aéreo y la alta velocidad ferroviaria: impactos sobre accesibilidad y medio ambiente (AERO-AVE)", concedido por el Ministerio de Fomento (Orden FOM/3836/2008), en la Convocatoria de 2008 de Ayudas a proyectos de I+D del Subprograma para la Movilidad sostenible y el cambio modal en el transporte. Acción Estratégica Energía y Cambio climático, en el marco del Plan Nacional de Investigación Científica, Desarrollo e Innovación Tecnológica 2008-2011.
} 


\title{
The air-rail intermodality of tourism in the city of Toledo
}

\begin{abstract}
With the opening of the new infrastructure in Madrid connecting the Atocha and Chamartin stations with the airport, the city of Toledo has become directly connected to the international airport of Madrid. This provides new possibilities for tourism due to the ability to attract international tourism to the city of Toledo.

Given the importance of connecting the international airport with the city of Toledo, together with the new trend of creating direct bus lines to the airport from different cities and the recent offers in combined TAV-Plan tickets for other routes, this study focuses on the intermodal mobility, in particular air-rail, of tourists visiting the city of Toledo.

The methodology is based on a descriptive analysis of the data obtained through a survey conducted in the corridor that allows an analysis to be made of the mode of transport used by tourists to access the city of Toledo, particularly from Madrid airport.
\end{abstract}

Keywords: intermodal tourism, mobility tourism, city of Toledo.

\section{INTRODUCCIÓN}

La intermodalidad aéreo-ferroviaria de viajeros como política de transportes se inicia a nivel europeo en el Libro Blanco de 2001 (Comisión Europea, 2001), que establece los principales beneficios del fomento de la intermodalidad para tratar de conseguir un transporte de pasajeros eficiente y sostenible con el medioambiente. Además, dada la situación actual y la tendencia de los gobiernos europeos a optimizar sus sistemas de transporte, es necesario centrar la atención en políticas que favorezcan una movilidad óptima y eficiente.

La intermodalidad aéreo-ferroviaria de pasajeros se puede definir como "un principio de política y de planificación cuyo objetivo es facilitar a los pasajeros, el uso de la alta velocidad ferroviaria y el transporte aéreo en un viaje sin ruptura de manera cómoda y que, como tal, contribuye a un sistema de transporte integrado y eficiente que establecerá redes interconectadas, donde los pasajeros disponen de más opciones de elección para sus viajes" (Nombela, 2011).

Los principales beneficios de la intermodalidad aéreo-ferroviaria son: la mejora de los niveles de congestión en las salidas y llegadas al aeropuerto, poder reducir las molestias causadas por el avión (ruido y emisiones), permite reducir costes operativos de las aerolíneas y aumentar el área de influencia de los aeropuertos (Eurocontrol, 2005b).

Por otro lado, las principales barreras para el desarrollo intermodal de los modos aéreo y ferroviario, que hacen que la competitividad del tren de alta velocidad sea más difícil de alcanzar son: las inversiones necesarias para el desarrollo de la infraestructura intermodal son muy costosas, la voluntad de los agentes en la coordinación o colaboración es limitada debido a aspectos de competencia, la falta de información a los pasajeros, las particularidades geográficas de los países y la percepción de los pasajeros sobre el transporte ferroviario es, en ciertos países, relativamente baja. (Eurocontrol, 2005b). 
Unido a estas barreras, la falta de interoperabilidad de los sistemas de transporte, es una de las causas del crecimiento del uso del vehículo privado en casi toda Europa (Eurocontrol, 2005a), lo que a su vez genera problemas de congestión del tráfico. La preferencia por el uso del vehículo privado se explica por su capacidad de proporcionar un modo de transporte "puerta a puerta". El objetivo de la intermodalidad es, por tanto, proponer también un trayecto "puerta a puerta" mediante la combinación de distintos modos de transporte, lo que permitiría reducir la congestión y los efectos negativos de la misma sobre el medioambiente.

La intermodalidad aéreo-ferroviaria es una meta planteada por la Unión Europea, que requiere de un conjunto de elementos perfectamente coordinados que permiten llegar a una plena integración del modo aéreo con el ferroviario, lo que exige grandes esfuerzos por parte de todos los agentes implicados.

Los factores o elementos que determinan la integración entre modos y que condicionan la oferta intermodal, van desde la existencia de una infraestructura de conexión adecuada hasta las cuestiones relativas a la manipulación de los equipajes o la seguridad. De todos los factores, el Libro Blanco de 2001 identifica como aspectos prioritarios en los que incidir en el corto plazo: el billete integrado, la gestión del equipaje y garantizar la continuidad de los desplazamientos (Comisión Europea, 2001). Posteriormente, en el Libro Blanco de 2011, la Comisión resalta como iniciativas para promover la movilidad "puerta a puerta" la confección de horarios, la información, los sistemas de reserva en línea y la expedición de billetes inteligentes (Comisión Europea, 2011). Otro aspecto a tener en cuenta es la promoción de la intermodalidad, que es necesaria para influir en el comportamiento del viajero, con el fin de aumentar la proporción de los viajes intermodales utilizando técnicas de sensibilización y gestión de la movilidad (Comisión Europea, 2004a).

Para su análisis estos elementos se agrupan en dos grandes categorías: factores referidos a la integración física, en los que encontraríamos las infraestructuras de conexión; y los referidos a la integración de servicios, en los que se encuentran, el tiempo de viaje y transbordo, los billetes y tarifas, la información, la manipulación del equipaje y la seguridad.

En cuanto a las infraestructuras, el concepto de intermodalidad tiene un papel clave en su planificación y permiten la combinación intermodal física. La planificación de las infraestructuras de transporte supone una barrera importante a la intermodalidad ya que generalmente no existen puntos de conexión entre los diferentes modos o estaciones intermodales, en concreto en los aeropuertos, donde los problemas de conexiones desde un punto de vista de la infraestructura estarían superados. Destacar en este estudio que de los distintos factores es necesario disponer de la infraestructura correspondiente y de una determinada calidad para poder articular cualquier política intermodal. Su grado de interconexión y la interoperabilidad física posibilita la intermodalidad y por tanto la integración de los distintos servicios de transporte, que aunque serán requisitos adicionales a la infraestructura, también resultan fundamentales para el pleno desarrollo de la intermodalidad.

Además de los elementos es necesaria una coordinación entre los agentes que intervienen en la intermodalidad, diferenciando entre los usuarios, los operadores y las autoridades implicadas. Cada uno de estos tiene una posición diferente ante cada elemento que debe ser analizada como factor condicionante de la intermodalidad. La colaboración resulta esencial para elaborar una oferta de servicios por parte de los operadores que, a 
menudo, es difícil debido a la desconfianza entre modos por ser potenciales competidores en otros corredores. Estas posiciones dependen fundamentalmente de los corredores a analizar, de forma que se diferencian tres tipos de situaciones: competencia, cooperación y complementariedad.

En este sentido, el ferrocarril y el avión han sido modos de transporte que han tenido una evolución desigual e inicialmente no han sido considerados competidores. Esta situación cambia por varios motivos: la liberalización del transporte aéreo con la aparición de las compañías de bajo coste y por la evolución tecnológica del transporte por ferrocarril, con la puesta en marcha del tren de alta velocidad (TAV).

Por tanto, en la situación actual, los operadores de estos modos tendrán un comportamiento en el mercado diferente y estará marcado en función de los corredores o rutas en los que operan. A partir de criterios de distancia y tiempo de viaje, y teniendo en cuenta el tipo de infraestructura aeroportuaria disponible diferenciamos dos tipos de rutas para la intermodalidad aéreo-ferroviaria, en términos de conexiones con un aeropuerto hub:

- De largo recorrido: rutas en las que se unen núcleos urbanos importantes, en los que normalmente coexisten ambos modos de transporte para la conexión de una ciudad con aeropuerto, con una metrópoli con aeropuerto hub con tiempo de recorrido en ambos modos de transporte de menos de tres horas.

- De corto y medio recorrido: rutas en las que se unen ciudades importantes con las ciudades cercanas y con las distintas provincias limítrofes, en las que normalmente no coexisten los modos de transporte. En algunos casos las ciudades disponen de ambas infraestructuras (aeropuerto y ferrocarril) pero no existen ofertas de rutas que compiten entre sí.

En el análisis de cada uno de los escenarios se encuentran tres situaciones: competencia, cooperación y complementariedad entre ambos modos de transporte en una ruta determinada (Cokasova, 2003). La competencia intermodal entre los modos aéreo y ferroviario se daría cuando existan distintas compañías que operan en un mismo corredor y por tanto coexisten ambos modos de transporte. La cooperación intermodal se daría en un corredor determinado, cuando exista un compromiso entre las compañías que operan en el mismo corredor en situación de competencia, donde normalmente uno de los modos (aéreo) dejará de operar en ese corredor mediante un acuerdo. La complementariedad intermodal se dará en los corredores donde no existe oferta aérea y el tren de alta velocidad facilita el acceso al aeropuerto.

Además con independencia del tipo de ruta en función de la distancia o el tiempo de viaje, hay que destacar que el transporte aéreo necesita de otros modos de transporte para que el pasajero acceda al aeropuerto, ya que normalmente estos se encuentran ubicados en la periferia de las ciudades. Los medios de acceder a los aeropuertos principales son el avión, el ferrocarril, el coche privado, el taxi y el autobús. En este sentido, para aprovechar las ventajas de la intermodalidad aéreo-ferroviaria, es muy importante que la conexión entre el transporte aéreo y el transporte terrestre sea a través de estaciones ferroviarias en los aeropuertos, que facilitan el acceso y evitan la congestión de las carreteras de acceso a los mismos, disminuyen los tiempos de viaje totales y tiene efectos beneficiosos para el medioambiente. 
La mayoría de las referencias en la literatura analizan las dos primeras tipologías, la competencia y/o la cooperación entre modos, olvidando aquellas rutas en las que tren y avión pueden complementar sus operaciones. Sólo los estudios que hacen referencia a la elección del aeropuerto son los que, normalmente, incluyen la elección del modo de acceso.

Del análisis de las principales experiencias intermodales en Europa se comprueba que aún quedan muchos hitos por alcanzar para lograr una integración modal que beneficie a todos agentes participantes, a los usuarios, a las empresas operadoras y a toda la sociedad en general.

En función de los criterios descritos anteriormente de los diferentes corredores en España, más concretamente en función del comportamiento de los distintos operadores a colaborar, a firmar acuerdos puntuales o a coexistir en el mismo mediante competencia intermodal, se plantean algunos ejemplos de rutas o corredores españoles donde existirá cooperación y competencia, como en el corredor Madrid-Barcelona o el Madrid-Málaga y complementariedad como en la ruta Madrid-Toledo.

Este último ejemplo, corredor Madrid-Toledo, es en el que se centra este análisis. Un corredor donde podrá existir complementariedad entre modos y por tanto intermodalidad aérea y ferroviaria en un contexto interurbano, en el que la ciudad de origen o destino no tiene aeropuerto sino que está dentro del área de influencia del aeropuerto de Madrid y se dará la situación de competencia intermodal en el acceso al aeropuerto.

Este corredor seleccionado cumple con el objetivo del estudio: la intermodalidad aéreo-ferroviaria en el caso de ciudades medias, que pertenecen al área de atracción de un gran aeropuerto y la influencia del acceso del TAV al aeropuerto, compitiendo en este ámbito, con el autobús y el vehículo particular. Para ello, se analizan los datos de una encuesta realizada en los meses de noviembre de 2010 a febrero de 2011, en la que se obtienen datos de preferencias reveladas y de preferencias declaradas. Los primeros analizan el perfil del viajero en la situación actual en el corredor Madrid-Toledo, mientras que en los segundos, se realiza un ejercicio de simulación para detectar las preferencias de los viajeros ante un escenario futuro de mejora de infraestructuras del transporte (corredor ferroviario Atocha-Chamartín-Aeropuerto) y de los servicios ofrecidos por los operadores (mejoras de la intermodalidad alta velocidad-aéreo).

Teniendo en cuenta la tendencia de nuevas líneas de autobuses directos al aeropuerto desde diferentes ciudades, unido a las recientes ofertas de billetes combinados TAV-Avión en otros corredores (Pérez, 2014), este estudio se centra en la movilidad intermodal, en concreto aéreo-ferroviaria, del turista que accede a la ciudad de Toledo.

\section{CARACTERÍSTICAS DEL CORREDOR TOLEDO-MADRID}

La evolución de la ciudad de Toledo está marcada por su carácter de ciudad histórica (declarada Patrimonio de la Humanidad), capital política y administrativa de la Comunidad de Castilla-La Mancha, centro de atracción turística a nivel nacional e internacional. Adicionalmente con el desarrollo de la gran conurbación madrileña, configura a Toledo como una ciudad media periférica de Madrid, con las correspondientes consecuencias en el proceso de desarrollo económico-urbano actual y de futuro.

La ciudad de Toledo cuenta con una población de 83.226 habitantes censada a 1 de enero de 2015. La población en el área de influencia, con un radio de 20 kilómetros, incluye 
12 municipios con una población de 132.522 habitantes. Si este radio de influencia se sitúa en 30 kilómetros, incorpora a 30 municipios, con una población que alcanza los 164.346 habitantes. La provincia de Toledo tiene una población de 693.371 habitantes, que recoge el 33,7\% de la comunidad autónoma de Castilla-la Mancha.

La superficie de la provincia de Toledo es de 15.370 kilómetros cuadrados que supone un $19,3 \%$ de la región. La densidad demográfica es de 45,1 habitantes por kilómetro cuadrado que representa un $174,1 \%$ de la densidad de la comunidad autónoma.

La ciudad de Toledo como capital turística ha registrado en el año 2015, 846.643 pernoctaciones, de las cuales el 68,8\% eran residentes en España y el 31,2\% no residentes. De los no residentes el 48,5\% provienen de países de la Unión Europea, principalmente de Francia, Reino Unido y Alemania; el $10,7 \%$ de Estados Unidos de América y destaca con un 6,4\% de Japón. La estancia media en 2015 ha sido de 1,5 días.

En el año 2015 se han registrado 564.558 viajeros, de los que el 68,7\% eran residentes en España y el 31,3\% no residentes. Los no residentes provenían de países de la Unión Europea el 47,0\%, de EE.UU el 10,4\% y el 8,6\% de Japón.

Respecto a la provincia de Toledo, según datos de la encuesta de ocupación hotelera (EOH) de 2015, la provincia registró la cifra de 762.435 viajeros, de los que el $27,4 \%$ eran no residentes, siendo la procedencia de los residentes en España principalmente de la Comunidad de Madrid en un 24,5\%, de la región en un 15,7\%, el 13,5\% de Andalucía y el $10,1 \%$ de la Comunidad Valenciana.

Los datos de las pernoctaciones de la provincia de Toledo son de 1.159.793 pernoctaciones según la EOH de 2015 , de los que el $72,8 \%$ son residentes en España y cuya procedencia es del 2,1\% de la Comunidad de Madrid, el 14,6\% de Castilla-La Mancha, 14,1\% de Andalucía y el $11,6 \%$ de Comunidad Valenciana.

Una de las características de la ciudad de Toledo es que es la sede del gobierno y del parlamento regional de Castilla-La Mancha, lo que implica la necesidad de movimientos interregionales de viajeros desde y hacia la ciudad.

La ciudad de Toledo puede definirse como una ciudad media, próxima a la gran área metropolitana de Madrid, que condiciona, hacia futuro el desarrollo territorial de la ciudad. Además, se plantean las bases de un gran eje de desarrollo territorial configurado por el corredor de Henares y el corredor de Toledo como el gran eje de desarrollo económico Guadalajara-Toledo-Madrid.

Este desarrollo territorial de Toledo ha de contemplar el área de influencia de la ciudad, incluir el conjunto de municipios en su entorno y definir políticas de desarrollo del territorio que contemplen el espacio económico más amplio, la ciudad de Toledo y su área metropolitana. En este sentido, es necesaria la creación de entes administrativos de coordinación (consorcios de servicios), algunos de ellos puestos en marcha (abastecimiento de agua, residuos, bomberos, etc.) y especialmente la creación de un consorcio de transportes, que permita promover acciones de fomento de la intermodalidad, tanto de mercancías como de pasajeros, nuevas infraestructuras del transporte (intercambiador Toledo) y el impulso de políticas de desarrollo territorial que afectan a las tres administraciones: locales, provinciales y regional. 
A continuación se muestran una selección de indicadores económicos sobre el empleo, las empresas, el comercio, la actividad comercial, nivel de motorización y el peso económico de la ciudad respecto a la provincia.

En términos de los indicadores, la provincia de Toledo presenta 202,2 miles de trabajadores afiliados a la Seguridad Social en el año 2015, lo que representa un 32,4\% de los afiliados en la comunidad autónoma. La distribución por sectores económicos es: 7,0\% en agricultura, $18,2 \%$ en industria, $8,3 \%$ en construcción, y $66,5 \%$ en servicios. De los trabajadores afiliados en la provincia de Toledo un $28,1 \%$ son trabajadores de alta en la ciudad de Toledo, de los que un 1,2\% pertenecen al sector de la agricultura, un $7,0 \%$ a la industria, un $3,1 \%$ a la construcción y un $88,8 \%$ al sector servicios.

El número de empresas inscritas en la Seguridad Social en el año 2015 en la provincia de Toledo era de 25.586, que recoge el 32,3\% de las inscritas en Castilla-La Mancha. Por sectores productivos tenemos que el $10,5 \%$ se dedican a la agricultura, el $11,3 \%$ a la industria, 9,0\% en construcción y $69,2 \%$ en servicios.

El producto interior bruto de la provincia de Toledo para el año 2013 es de 11.542 millones de euros que es un $31,0 \%$ del total de la región. La estructura productiva en la que se distribuye este PIB es, un 6,3\% en agricultura, $21,0 \%$ en industria, 6,0\% en construcción y $58,1 \%$ en servicios y $8,7 \%$ impuestos netos sobre producción.

En cuanto al comercio, la ciudad de Toledo dispone de cinco centros comerciales con una superficie bruta de 123.950 metros cuadrados. Cercanos al municipio se encuentra un centro comercial mediano (Olías del Rey) y un nuevo centro comercial grande inaugurado en 2011 (Bargas).

La actividad comercial en la provincia de Toledo a nivel mayorista presenta un conjunto de 2.327 establecimientos, en los que Toledo representa el 10,1\%. A nivel minorista, la provincia dispone de 11.229 establecimientos de los que la ciudad incorpora $15,6 \%$ del total.

Otro indicador económico es el nivel de motorización que en la provincia de Toledo, es de 355,6 automóviles por cada 1000 habitantes. Para la ciudad de Toledo el nivel de motorización es de 41,2 automóviles por cada 1000 habitantes, con niveles importantes de motorización en los municipios del área de influencia (Olías del Rey, Nambroca, Cobisa, etc.).

\section{METODOLOGÍA}

El estudio del corredor Toledo-Madrid se basa en el análisis de los datos de una encuesta realizada sobre la movilidad del corredor Madrid-Toledo, siguiendo la metodología de captación de datos de Preferencias Reveladas (PR) y Preferencias Declaradas (PD). La encuesta de PR trata de obtener información, sobre el tipo de movilidad de los viajeros en la situación actual (perfil socio-económico, parámetros de movilidad) y la encuesta de PD plantea, un ejercicio de simulación de escenarios alternativos que configuran la movilidad en el futuro.

La encuesta se plantea con el objetivo de estudiar las características de movilidad en el corredor Toledo-Madrid y Madrid-Toledo. Para ello se diferenciaron dos perfiles de viajeros en función del motivo de movilidad: viajeros cuyo perfil de movilidad es obligado (trabajo o estudios/formación) y para el viajero que visita Toledo por motivo no obligado 
(ocio o turismo). Además, para detallar el esquema de movilidad con intermodalidad aéreoferroviaria del corredor se detectó aquel individuo que estaba realizando en ese momento o que había realizado en alguna ocasión el trayecto completo, para captar al viajero turista que viene directamente a Toledo a través del aeropuerto de Madrid.

Por tanto, de esta encuesta se obtienen dos líneas de investigación: en primer lugar medir la movilidad en el corredor y en segundo lugar, analizar la captación de usuarios por parte del ferrocarril (tren de alta velocidad), procedentes de los otros modos de transporte (vehículo privado y autobús), por una mejora en la intermodalidad derivada de la infraestructura de inter-conexión entre la red de alta velocidad y el aeropuerto de Madrid.

La muestra recoge los viajeros del corredor Toledo-Madrid y Madrid-Toledo, para tratar de evidenciar cuál es la cuantía que realiza el trayecto a través del aeropuerto de Madrid del total de usuarios que se mueven por este corredor diariamente. En este punto resulta importante resaltar que debido a las características del corredor, por su reducida distancia entre estos puntos, resultó una labor muy difícil captar al pasajero que realizaba o había realizado alguna vez este recorrido (Toledo-Madrid-Aeropuerto).

El trabajo de campo se llevó a cabo mediante una encuesta de tipo personal realizada entre los meses de noviembre y diciembre de 2010, y de tipo "on-line" durante los meses de diciembre de 2010 a febrero de 2011. Se completaron un total de 1.514 encuestas válidas a usuarios del corredor Madrid-Toledo, para los diferentes modos y motivos de movilidad del viaje.

Centrados en el perfil del viajero que llega a la ciudad de Toledo por motivos de esparcimiento tales como ocio y/o turismo, las entrevistas se realizaron en puntos estratégicos de llegada de turistas, como las estaciones de ferrocarril y autobuses de Toledo y Madrid y en diferentes puntos de información turística de la ciudad. Las preferencias reveladas se plantearon a todos los usuarios de los distintos modos de transporte utilizados en el trayecto Toledo-Madrid-Aeropuerto, estableciéndose un cuestionario único para todos los modos de transporte disponibles para realizar el trayecto (tren de alta velocidad, autobús y vehículo particular). Los cuestionarios se estructuraron agrupando las preguntas en función de los dos bloques temáticos: características socioeconómicas y características de la movilidad. Las preferencias declaradas, al igual que las anteriores, se plantearon a todos los usuarios de los tres modos de transporte disponibles en dicho recorrido.

En este proceso se siguen los siguientes pasos (Martínez y Muro, 2011):

- Análisis socioeconómico en el que se caracteriza a la ciudad de Toledo y su entorno metropolitano (de entre 20 a 30 kilómetros), mediante indicadores socioeconómicos.

- Análisis de la oferta para la determinación de la oferta actual disponible para todos los modos de transporte del corredor (precios, frecuencias, etc.).

- Análisis de la demanda actual referida a la demanda anterior a la realización de la encuesta de los modos de transporte disponibles (número de viajeros de cada modo de transporte).

- Análisis de los resultados de la encuesta que se divide en dos resultados: el análisis descriptivo de los datos y la modelización de los resultados de las preferencias declaradas. 
Estos análisis son posibles gracias a la obtención de los dos tipos de datos ya mencionados: de preferencias reveladas que tratan de obtener información sobre el tipo de movilidad de los viajeros en la situación actual (perfil socio-económico y parámetros de movilidad) y de preferencias declaradas en el que se plantea un ejercicio de simulación de escenarios alternativos que configuran la movilidad en el futuro, basada en este caso en la nueva infraestructura de conexión con el aeropuerto de forma que plantean diferentes opciones de servicio, que se basan en un recorrido total con dos transbordos, con un transbordo o ningún transbordo.

Tabla 1. Ficha Técnica de la encuesta.

El tamaño de la muestra en cada modo de transporte se determinó teniendo en cuenta la información disponible en los estudios previos, llevados a cabo, acerca de la distribución modal en el corredor.

Público objetivo: Personas mayores de 18 años que hayan tenido que desplazarse en alguna ocasión a Madrid y/o al aeropuerto de Madrid, desde la ciudad de Toledo.

Tamaño de la Muestra Total: 1514 casos, lo que conlleva un error teórico de muestreo del $\pm 2,6 \%$ al nivel de confianza del 95,5\% ( $p=q=0,5)$.

Distribución Muestral: Estratificado por modos de transporte: TAV, autobús y coche, por motivo de movilidad: OBLIGADO y NO OBLIGADO.

Tipo entrevista: Encuesta personal, a través de cuestionario, con una duración aproximada de 15 minutos, a pie de calle y en estaciones de autobús y TAV, empresas y centros de afluencia de turismo.

Fecha de realización del trabajo de campo: Entre el 10 de noviembre hasta el 13 de diciembre de 2010 para la encuesta presencial y entre el 30 de noviembre de 2010 hasta el 1 de febrero de 2011 para la online.

Errores de Muestreo: De acuerdo con la distribución de la muestra, según modos de transporte: TAV, Autobús y Coche y según motivo de movilidad: OBLIGADO y NO OBLIGADO, se derivan los siguientes errores de muestreo en cada conglomerado:

Motivo Obligado: Muestra de 1.017 unidades muestrales, extraídas por muestreo aleatorio simple, con afijación proporcional. Nivel de confianza 95,5\%. Error de muestreo: 3,0\%.

Motivo No Obligado: Muestra de 497 unidades muestrales, extraídas por muestreo aleatorio simple, con afijación constante. Nivel de confianza $95,5 \%(p=q=0,5)$. Error de muestreo: $4,2 \%$.

\section{Elaboración propia.}

\section{RESULTADOS DESCRIPTIVOS}

El motivo de movilidad no obligada, de visita y/o turismo será aquel realizado por razones de viaje no obligado, tales como vacaciones, turismo y ocio de fin de semana. En estos casos, Toledo es un destino destacable para los viajeros que llegan a la capital regional. Su atributo como Ciudad Patrimonio de la Humanidad y su atractivo inalterable a través de los años consiguen que esta ciudad sea de paso obligado para los visitantes. De este modo, analizar los medios de transporte utilizados por los turistas para llegar a Toledo y el por qué eligen estos medios, es de gran relevancia para intentar plantear nuevas formas de transporte y, en definitiva una oferta más cómoda, económica, y por supuesto, sostenible a nivel medio ambiental.

En primer lugar se describe el perfil socioeconómico del viajero del corredor Madrid Toledo como el de un hombre (55\%) de entre 18 a 33 años (41\%). 
Gráfico 1. Sexo y edad por movilidad no obligada

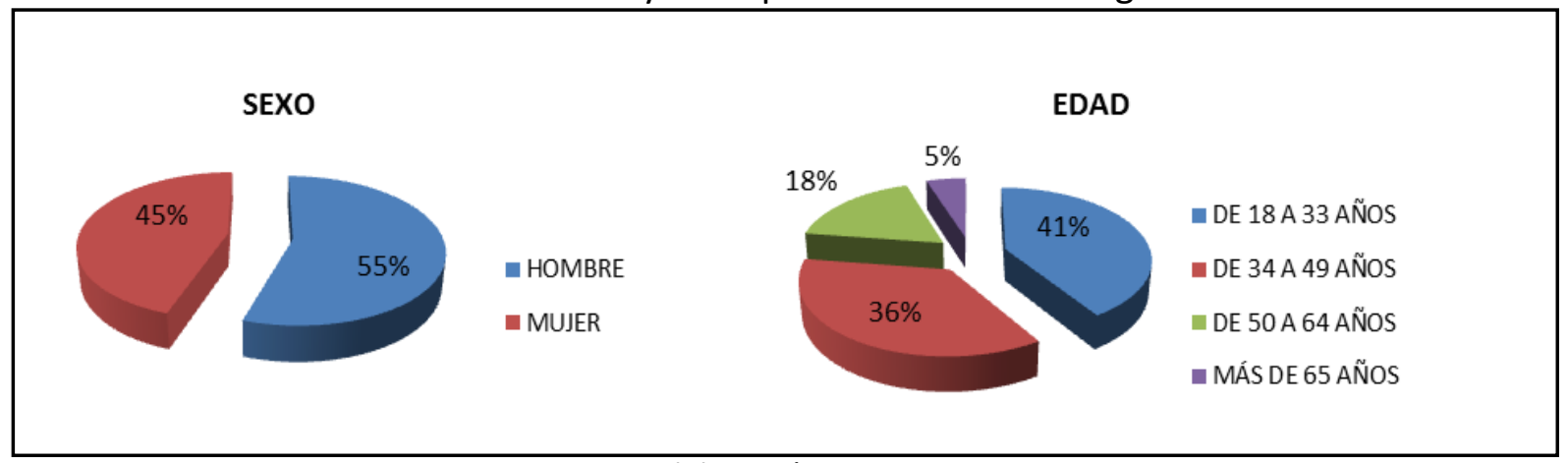

Elaboración propia.

La actividad profesional principal del viajero, que muestra el Gráfico 2, es en su mayoría trabajadores por cuenta ajena (66\%), de los cuales muchos trabajan en una empresa privada (44\%) y para la administración pública (22\%). El rango salarial que destaca es el de 1.001 a 2.000 euros (44\%).

Gráfico 2. Actividad profesional y nivel salarial por movilidad no obligada

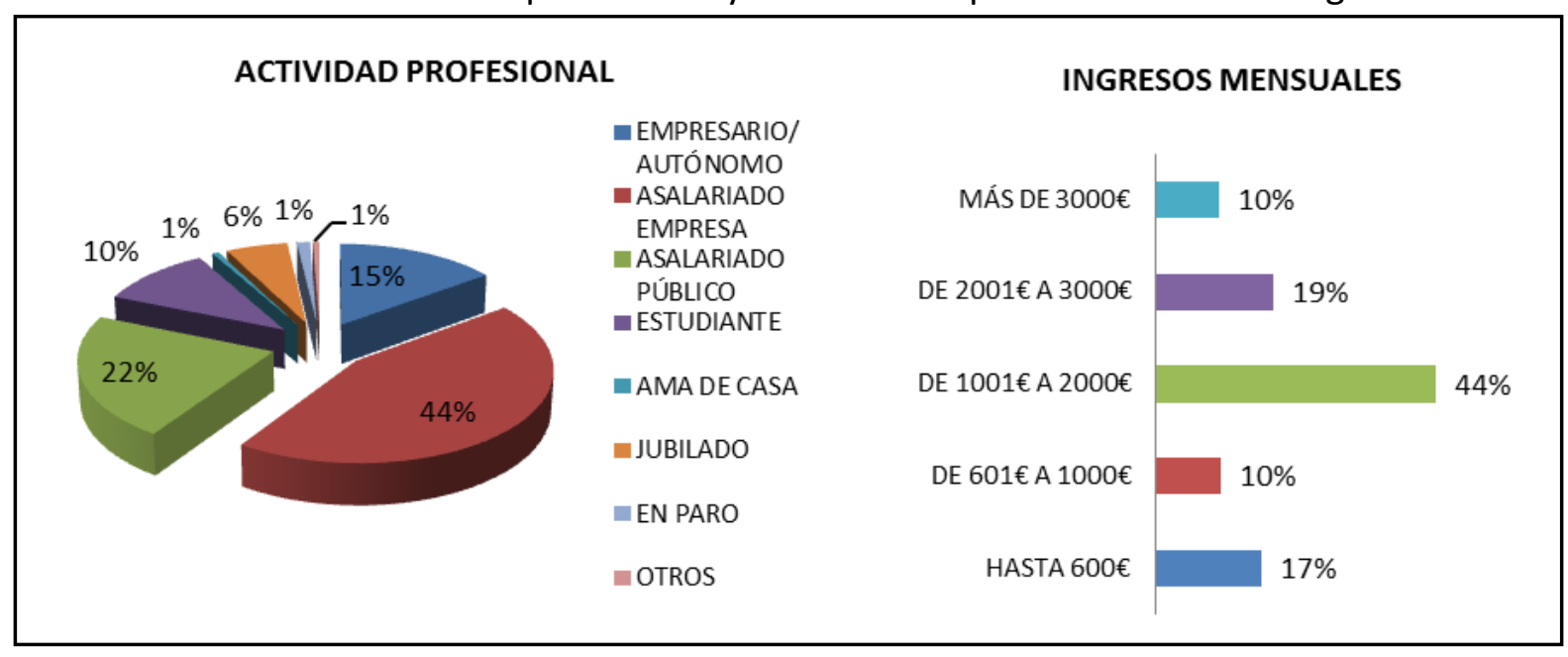

Elaboración propia

En cuanto al destino de los viajeros, Toledo ocupa en sus vacaciones el primer y único destino de los visitantes (56\%), seguidos de los viajeros cuyo destino es intermedio en su ruta $(25 \%)$ siendo menor el porcentaje de primer destino que va a visitar durante sus vacaciones (11\%). 
Gráfico 3. Origen del viaje y lugar que ocupa Toledo por movilidad no obligada

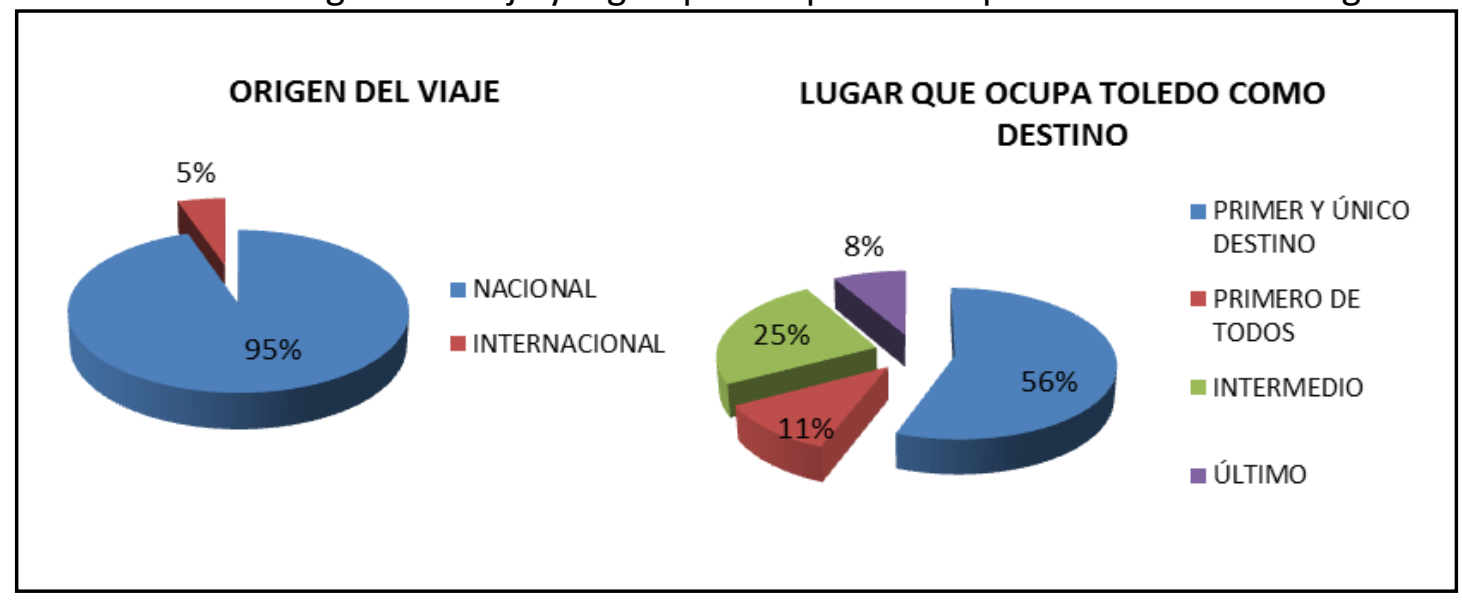

Elaboración propia

El origen principal de los visitantes a Toledo es de otros municipios de España (95\%), destacando que el mayor volumen procede de Madrid (68\%). Otro grupo importante es el que viene desde otras provincias cercanas a Toledo y de pueblos de la provincia de Toledo (22\%).

En el Gráfico 4 se muestra el origen internacional (5\%) por países, donde principalmente los viajeros pertenecen a países situados en Centro Europa (39\%), seguidos muy de cerca por aquellos que visitan Toledo desde algún lugar de Asia (34\%).

Gráfico 4. Origen nacional e internacional por movilidad no obligada

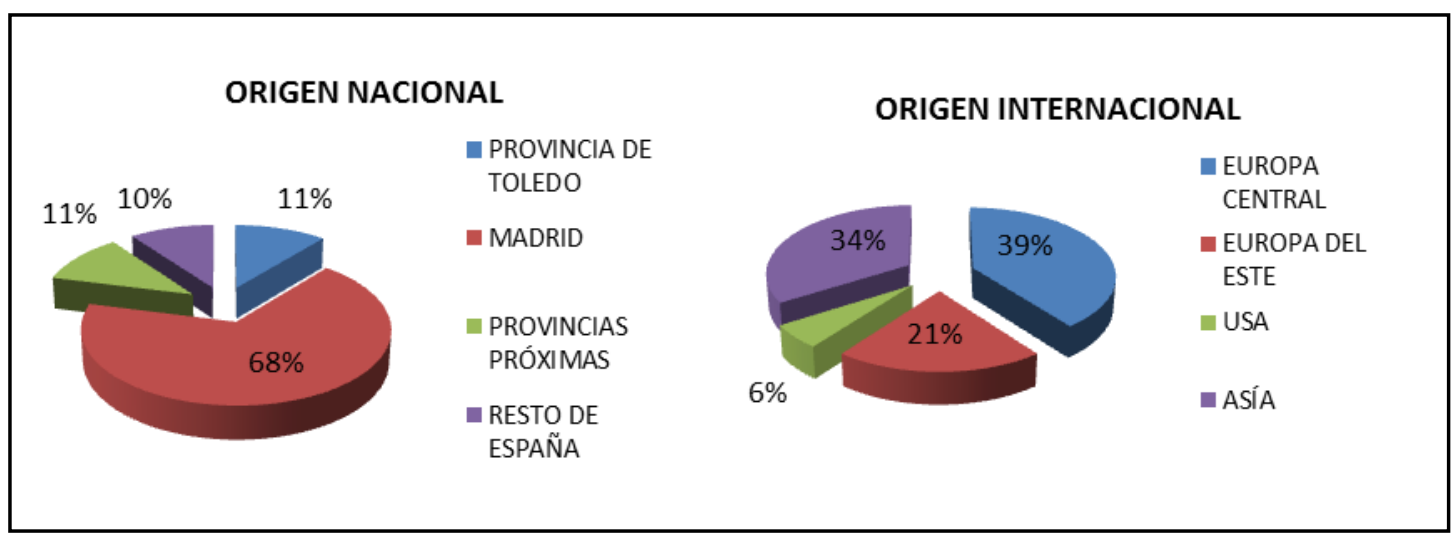

Elaboración propia.

Un factor importante para estudiar la intermodalidad aéreo-ferroviaria, es conocer los modos de transporte utilizados por los turistas para acceder desde su origen hasta Toledo y comprobar qué frecuencia de uso tiene cada uno de ellos. El Gráfico 5 muestra que con mayor frecuencia el turismo llega en coche (60\%), seguido del tren (30\%) y del autobús (10\%). De los usuarios del vehículo privado la titularidad es propia en su gran mayoría (92\%), seguida del vehículo alquilado (5\%) y en su mayoría no utilizaron la autopista de peaje $(80 \%)$. 
Gráfico 5. ¿Cómo llegó a Toledo?

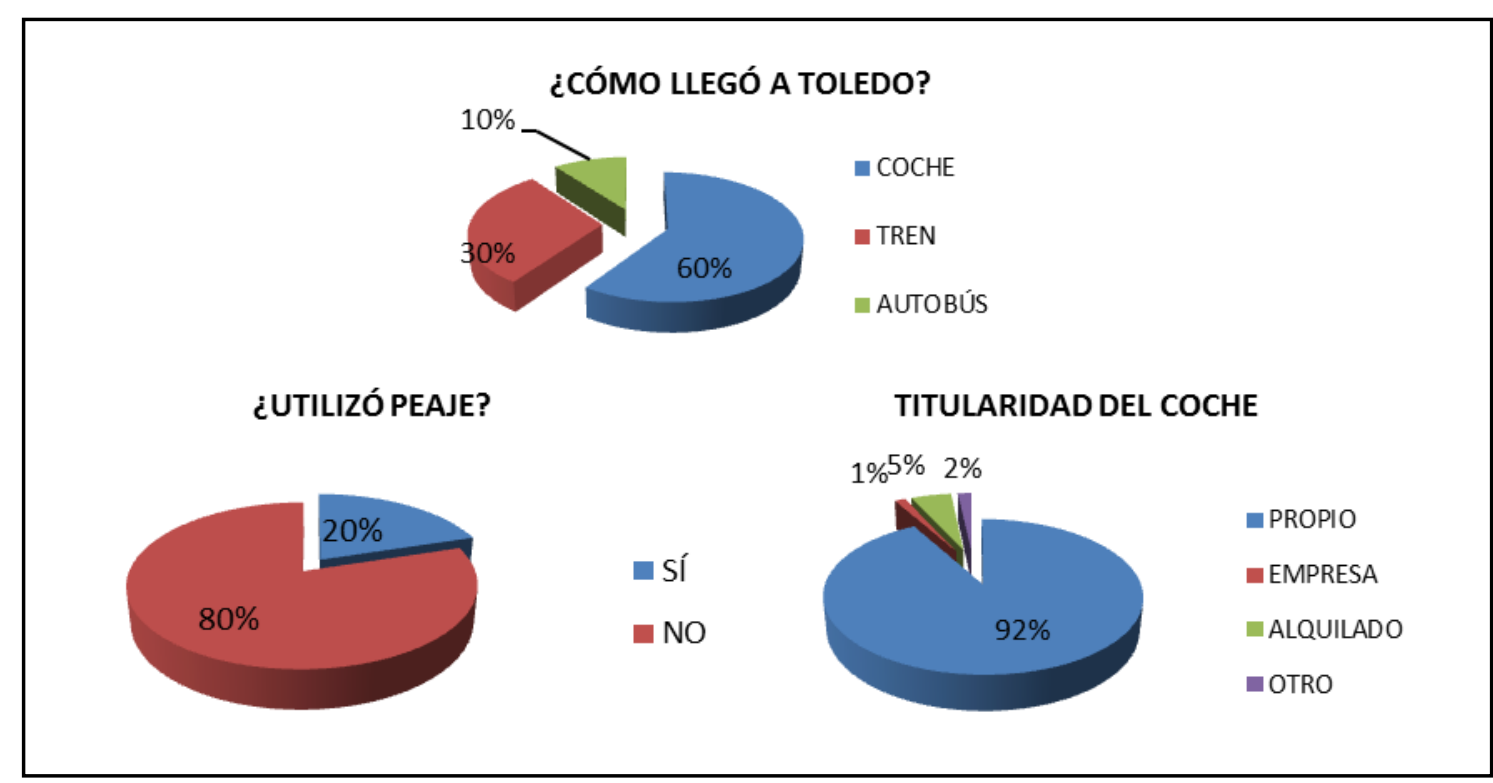

Elaboración propia

El acceso desde el aeropuerto de Madrid a Toledo (Gráfico 6) es minoritario respecto del total de los encuestados (27\%), de los cuales la mayor parte son usuarios de los modos aéreo y ferroviario, y por tanto potenciales usuarios de la mejora de la intermodalidad, en el (59\%). El resto de los viajeros llega a Toledo desde el aeropuerto de Madrid en autobús (21\%) y en coche (20\%).

Gráfico 6. Acceso y modo de acceso a Toledo por el aeropuerto de Madrid

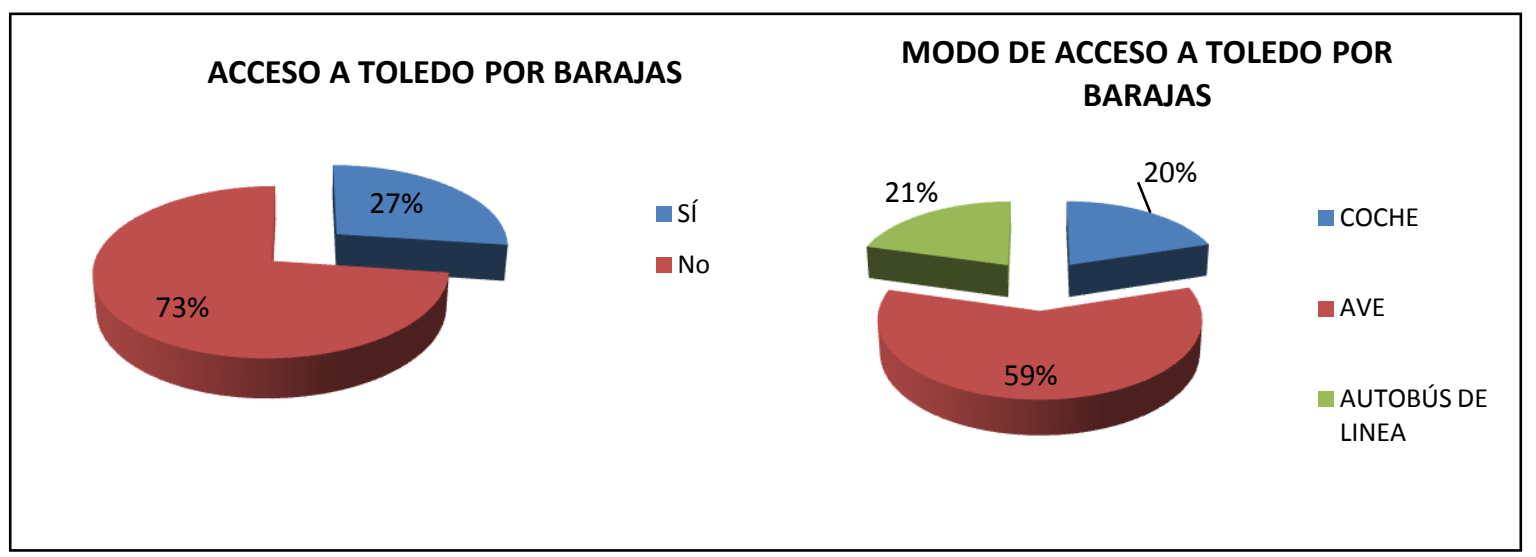

Elaboración propia.

El Gráfico 7 muestra el modo de acceso desde otros puntos diferentes al Aeropuerto (73\%), siendo el coche (75\%) y el TAV (19\%) los predominantes. 
Gráfico 7. Medio de transporte para llegar a Toledo por movilidad no obligada.

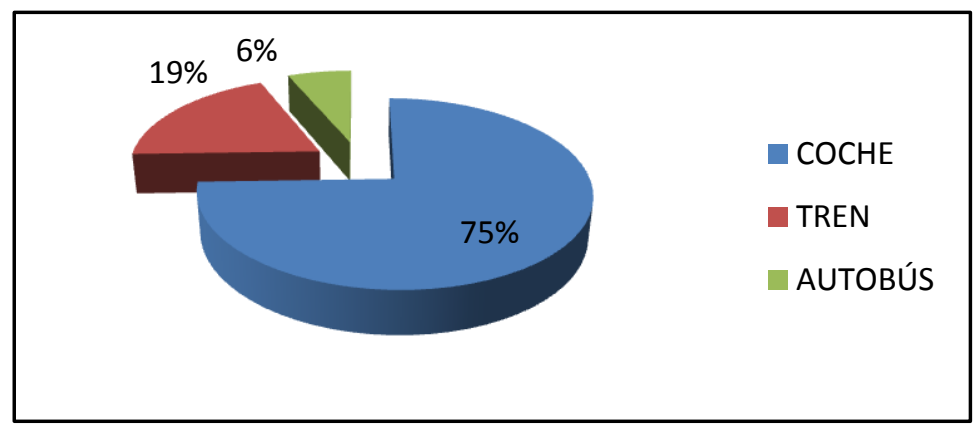

Elaboración propia

En cuanto a la duración total de las vacaciones es en su mayoría entre 1 a 2 días (55\%), lo que condiciona la estancia en la ciudad, ya que generalmente no pernocta en Toledo (64\%).

Gráfico 8. Duración de las vacaciones y estancia en Toledo

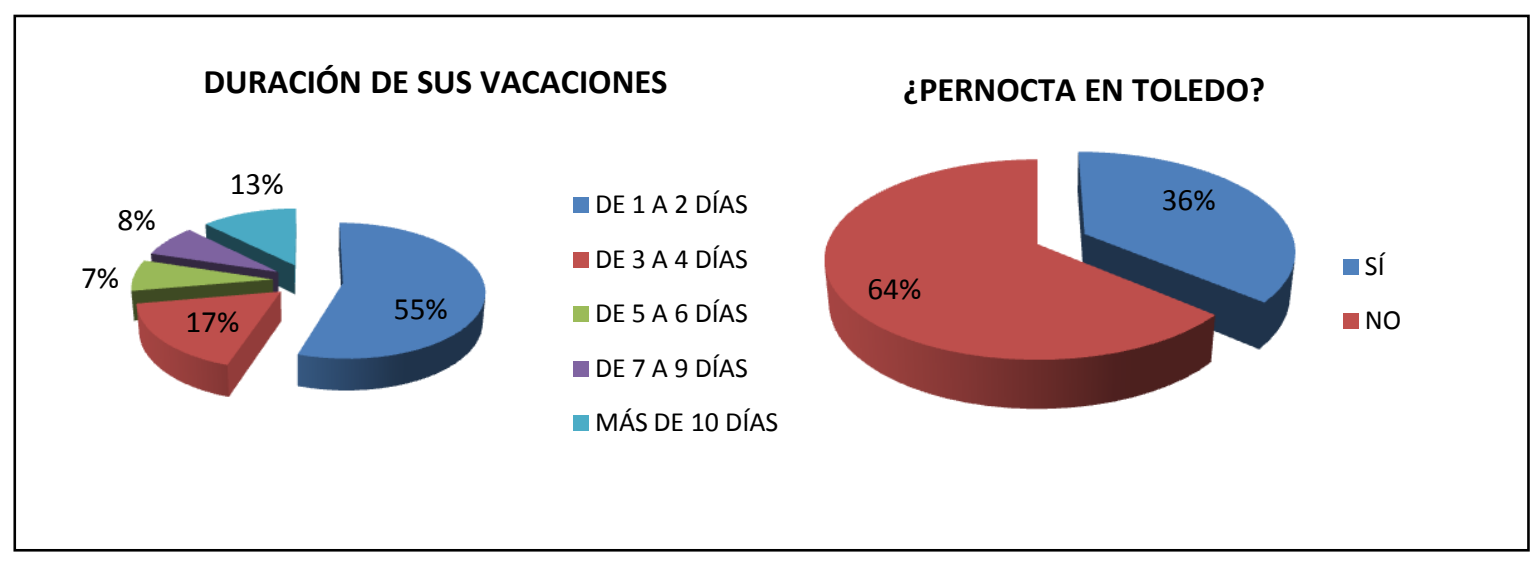

Elaboración propia

De los turistas que pernoctan en Toledo (36\%), en su mayoría lo hacen por una noche (37\%) o dos noches (47\%) y es poco frecuente más de tres noches $(5 \%)$ y de los que deciden pasar al menos una noche en la ciudad de Toledo, podemos observar en el Gráfico 9 que la mayoría lo hace en un hotel de tres estrellas o más (67\%). 
Gráfico 9. Número de pernoctaciones y tipo de alojamiento

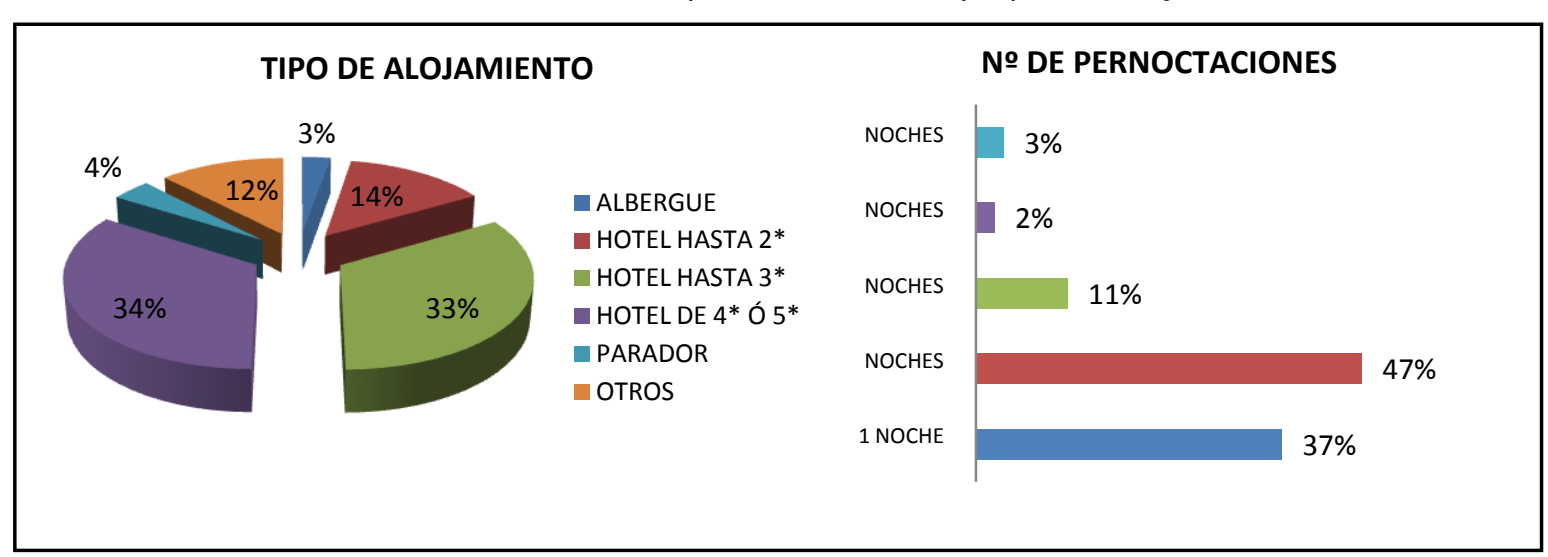

Elaboración propia

La planificación y organización del viaje del turista (Gráfico 10) desde la perspectiva de la forma de organizar el viaje respecto a la compra del título de transporte es mayoritariamente un billete individual (91\%) y se adquiere el mismo día (58\%), siendo el lugar de compra la taquilla de la estación (74\%), seguido de internet (16\%). Generalmente los viajeros compran un billete de ida y vuelta (85\%), cuyo descuento se aplica al billete de vuelta si este se deja abierto y dado que los viajeros son encuestados a su llegada a Toledo, la tarifa de descuento predominante es la de jubilado/pensionista (7\%). 
Gráfico 10. Planificación y organización del viaje

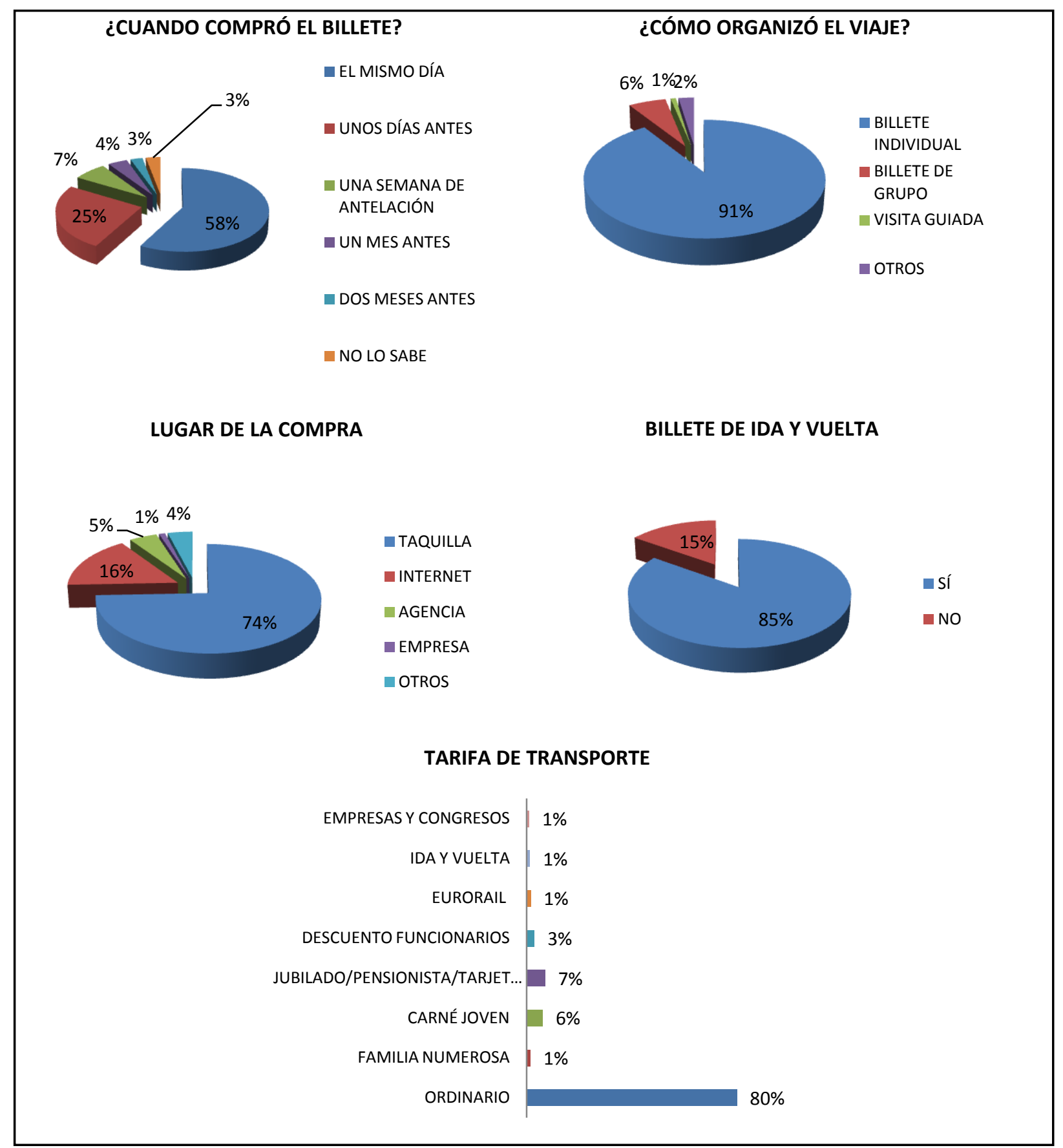

Elaboración propia. 
La motivación de la elección de los modos de transporte de los viajeros que llegan a Toledo por turismo es la comodidad del medio. Se observa en el Grafico 11 que la elección del viajero se basa fundamentalmente en la comodidad que le proporciona el TAV (75\%), seguida muy de lejos por la variable rapidez (11\%). Aunque, en cuanto a la comodidad sigue ganando el coche como cualidad destacada para los viajeros del mismo (88\%), mientras los viajeros del autobús destacan que lo eligen por ser el más barato (37\%).

Gráfico 11. Principales motivos de elección del modo de transporte y motivos de elección por el modo de transporte utilizado

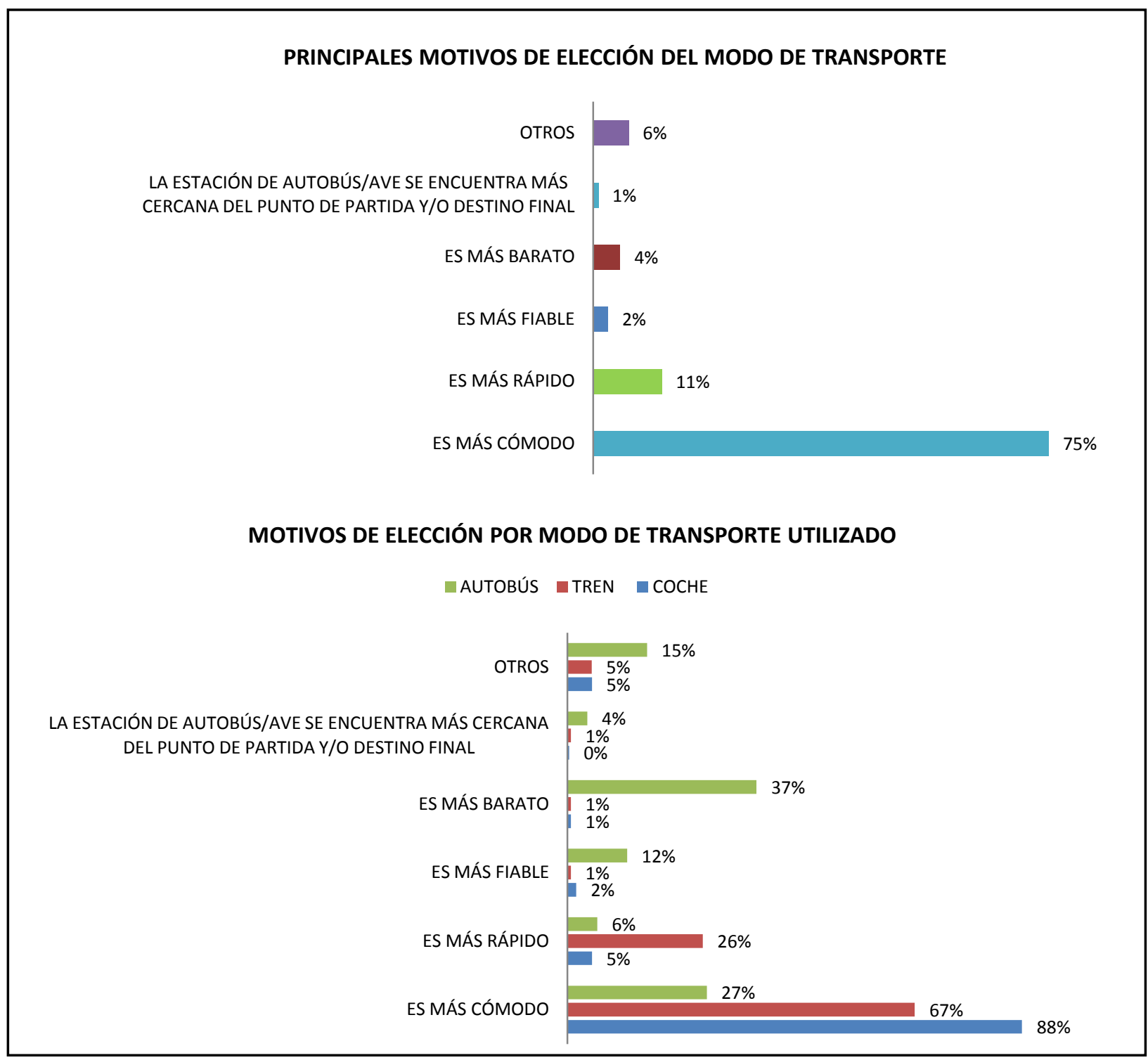

Elaboración propia.

Para finalizar el análisis descriptivo de los datos obtenidos en la encuesta y aunque la utilización más destacable de los datos de preferencias declaradas es la modelización de la demanda de transportes, vamos a realizar un análisis descriptivo previo que permitirá conocer en profundidad los datos disponibles y detectar posibles errores en los mismos, lo que nos sirve para depurar la base de datos. 
La metodología básica para el planteamiento de las opciones de las preferencias declaradas (PD) es la realización de un diseño eficiente de los escenarios. En concreto el cuestionario de preferencias declaradas se diseña siguiendo un esquema de disminución de tiempos y precios, en tres bloques o escenarios, para determinar la variación de la elección de los individuos.

Las encuestas de PD se estructuran con dos planteamientos: un primer planteamiento comparativo entre el autobús y el TAV y un segundo planteamiento comparando el coche y el TAV. Los escenarios planteados se conforman con las variables: precio del viaje, tiempo del viaje, comodidad y coste de aparcamiento, esta última variable referida solo al caso del coche.

Las variables tiempo, precio y costes de aparcamiento que sirven de base para el establecimiento de los escenarios planteados son los obtenidos teniendo en cuenta las distintas alternativas de viaje actuales en el corredor Toledo-Madrid-Aeropuerto. A continuación se selecciona la ruta óptima en tiempo y precio para el caso de las tres alternativas de elección (autobús, coche y TAV). La alternativa óptima actual del TAV escogida en el paso anterior servirá de base para calcular las variables de los escenarios que se han supuesto respecto a la utilización de la futura nueva infraestructura TAV/Cercanías que une Atocha con el aeropuerto de Madrid.

En consecuencia se establecen tres escenarios de trayectos que llevan implícitos unos tiempos y unos precios de viaje:

- Toledo-Aeropuerto sin transbordo: Utilización de la nueva infraestructura, realizando el viaje en TAV desde Toledo hasta el Aeropuerto de Madrid haciendo dos paradas, una en la estación de Atocha y otra en la estación de Chamartín pero sin realizar ningún transbordo.

- Toledo-Aeropuerto con un transbordo: Utilización de la nueva infraestructura, realizando el viaje en TAV desde Toledo hasta el Aeropuerto de Madrid haciendo una parada en la estación de Atocha y otra parada con transbordo en la estación de Chamartín.

- Toledo-Aeropuerto con dos transbordos: Utilización de la nueva infraestructura, realizando el viaje en TAV desde Toledo hasta el Aeropuerto de Madrid realizando una parada con transbordo en la estación de Atocha y otra parada con transbordo en la estación de Chamartín.

Las alternativas óptimas elegidas de los modos autobús y coche son fijas y comparadas en bloques de tres, de acuerdo con los tres escenarios planteados para el TAV. Los precios y los tiempo de viaje para el modo de transporte autobús y vehículo privado son constantes para todos los escenarios y están basados en los valores actuales. Los precios del autobús están calculados en función de los precios de los billetes y los precios del vehículo privado se calculan aplicando un coste por kilómetro recorrido que incorpora el mantenimiento del vehículo, seguros, etc., sumando el coste del peaje. Este precio no incorpora coste del parking ya que se presenta como una variable diferente medido en euros por día de parking.

Para detectar comportamientos cautivos, lexicográficos y de inercia, en las elecciones de los individuos se analizan las bases de datos en función de si cada uno de ellos ha variado 
o no la elección de la alternativa en los distintos escenarios a lo largo del ejercicio planteado. Los individuos cautivos son aquellos que eligen siempre la misma opción independientemente de las alternativas que se les presenten, bien por inercia o bien porque no tienen disponibilidad del modo ${ }^{2}$. Los individuos lexicográficos, son aquellos que eligen una opción de acuerdo a una característica en la que esta alternativa es superior, por ejemplo, el individuo que siempre elige la alternativa que tiene un menor coste independientemente del resto de los atributos. La inercia del individuo es la tendencia de elegir la opción que utilizan habitualmente en la actualidad y no cambian.

A continuación se muestra un análisis descriptivo de las encuestas de preferencias declaradas:

- Las frecuencias resultantes de elección de cada alternativa son de 382 encuestados referidos a la movilidad no obligada y se obtienen 3.438 observaciones ${ }^{3}$.

- La elección de la alternativa TAV está formada por 2.436 observaciones (70,9\%); 1.079 de la alternativa autobús $(17,7 \%)$ y 343 de la alternativa coche $(11,4 \%)^{4}$.

Tabla 2. Resumen número de elecciones por alternativa

\begin{tabular}{|l|l|c|c|}
\hline \multicolumn{4}{|c|}{ Base motivo de movilidad no obligado (DB_TUR) } \\
\hline TAV & BUS & COCHE & TOTAL \\
\hline 2.436 & 609 & 393 & 3.438 \\
\hline \multicolumn{4}{|c|}{ Total encuesta } \\
\hline TAV & BUS & COCHE & TOTAL \\
\hline 7.059 & 1.688 & 736 & 9.483 \\
\hline
\end{tabular}

Elaboración propia

- Los encuestados que siempre eligen las mismas alternativas en los diferentes escenarios planteados y en cada una de las tres opciones son:

- Para la alternativa TAV: son 219 individuos, por tanto 1.971 observaciones los que siempre eligen $\mathrm{TAV}^{5}$, independientemente del precio menor del autobús o del menor tiempo del coche.

- Para la alternativa AUTOBús: los que siempre eligen la alternativa autobús son 38 individuos con sus respectivas 342 observaciones. Esto son todos aquellos individuos que priman el precio independientemente del tiempo de viaje.

- Para la alternativa $\mathrm{COCHE}^{6}$ : siempre eligen la alternativa COCHE, 25 individuos, lo que suponen 225 observaciones. La elección de esta alternativa supone tiempos de viaje menores respecto al TAV.

\footnotetext{
${ }^{2}$ En este estudio se supone que todos los encuestados tienen disponibles todas las alternativas.

${ }^{3}$ Por cada individuo se obtienen nueve respuestas sobre los tres escenarios (tres alternativas de elección por escenario planteado).

${ }^{4}$ El menor número de observaciones que ha elegido en alguna de las opciones el coche mejora frente a la base obligada, porque en este caso, se capta al potencial usuario, debido a la ampliación de lugares donde se realizó la encuesta (oficinas de información turística en Toledo) y que enriquecen la muestra.

${ }^{5}$ En esta base de datos se advierte un mayor número de elecciones de TAV ya que las alternativas que se presentan son el TAV, frente autobús y al coche.

${ }^{6}$ En el caso de la movilidad no obligada la alternativa de elección se refiere al coche alquilado. 


\section{CONCLUSIONES}

El corredor Toledo-Madrid-Aeropuerto es un ejemplo potencial de la intermodalidad de tipo complementariedad intermodal en un contexto de entorno interurbano de corta distancia y con peculiaridades que afectan al tipo de movilidad de pasajeros. En concreto, las características de la ciudad de Toledo son:

- Ciudad media con potencial desarrollo económico y territorial con la configuración del área metropolitana de Toledo.

- Ciudad de atracción turística reconocida a nivel nacional e internacional.

- Proximidad a la gran área metropolitana de Madrid y el consiguiente desarrollo del eje económico Madrid-Toledo.

Estas características unidas a la aparición de la nueva infraestructura de transporte que conecta la red de alta velocidad con el aeropuerto de Madrid (corredor ferroviario Atocha-Chamartín-Aeropuerto), posibilita la mejora en la oferta de los servicios de los diferentes operadores modales, mediante diferentes figuras de títulos de transporte, tiempos de conexión, gestión del equipaje, visualización de la información y la incorporación de las tecnologías TIC a la operativa del transporte (integración de sistemas de información y reserva).

Ante este escenario, con el estado actual de la movilidad, teniendo en cuenta los previsibles cambios en la mejora de la intermodalidad entre el modo aéreo y el tren de alta velocidad, se detectan siguientes las barreras a la intermodalidad:

- Escaso fomento de la intermodalidad por parte de los operadores aéreos y ferroviarios, sin tener ningún tipo de convenio de cooperación.

- El desconocimiento de los usuarios de este corredor.

- Los hábitos y costumbres de los usuarios del vehículo privado.

Por todo ello, existe una muy débil intermodalidad por parte de la demanda ya que la oferta intermodal es inexistente y por tanto, no hay colaboración entre los agentes implicados, unido a que son muy pocos usuarios los que en la actualidad realizan este trayecto utilizando ambos modos.

El análisis de los datos de la encuesta describe un perfil socio-económico y de movilidad del turista que llega a Toledo, como un individuo (hombre/mujer) de entre 18 a 33 años que trabaja por cuenta ajena con un nivel salarial de entre 1.001 a $2.000 €$ y cuyo origen del viaje es en su mayoría nacional en concreto de Madrid, por lo que llega a la ciudad en vehículo particular y no llega a pernoctar en Toledo. Los viajeros que proceden del aeropuerto que llegan a Toledo lo hacen, principalmente, con el TAV ${ }^{7}$. Las preferencias de la mayoría de los encuestados es la de realizar la combinación del TAV y el avión si existiera una oferta conjunta mediante la cooperación entre modos con una clara preferencia del modo TAV-Avión, frente al resto de las alternativas planteadas en el recorrido MadridToledo.

\footnotetext{
${ }^{7}$ Los resultados del análisis descriptivo muestran los problemas que condicionarán la utilización de estos datos, ya que las muestras que se han obtenido para el caso de los viajeros que realizan o han realizado en alguna ocasión el trayecto Madrid - Aeropuerto de Madrid representan un 18,8\% de la muestra total, lo cual determinará los resultados de la modelización. Investigaciones Turísticas 
Una política de transportes enfocada a sustituir el transporte privado por el transporte público (TAV), beneficia por un lado los usuarios que tendrán menores tiempos de viaje y en general, al sistema de transportes por disminuir la congestión de los accesos a Madrid y al Aeropuerto de Madrid. Las principales variables para fomentar la integración de los modos de transporte aéreo y de alta velocidad son la visibilidad de la oferta, la gestión integrada de la reserva y tarjetas de embarque, el check-in y control de equipaje, la responsabilidad del viaje completo, la gestión de los programas de fidelización de viajeros, la facilidad de conexión en el modo intermodal, el tiempo de viaje y el precio (Muro, 2012). En cuanto a esta oferta, y sobre todo referido a las conexiones con el aeropuerto, han surgido nuevas líneas de autobuses directos al aeropuerto desde diferentes ciudades y ofertas de billetes combinados TAV-Avión en otros corredores, lo que indica mejoras en la intermodalidad que podrían ser aplicables en un futuro al caso que nos ocupa.

Para finalizar se pueden diferenciar dos movimientos de los viajeros de Toledo, de entrada y de salida, que permiten distinguir tipos de demanda: la intermodalidad de demanda de salida de Toledo que fundamentalmente es por motivo obligado. $\mathrm{Y}$, por otro lado, la intermodalidad de demanda de entrada en Toledo que es principalmente por motivo de turismo. En este caso, con el análisis de demanda actual y de oferta del turismo en la ciudad de Toledo, se detecta que hay un segmento del turismo que habría que analizar detalladamente y que no se ha recogido en la encuesta y por tanto en este análisis. En este segmento se encuentran todos aquellos turistas/visitantes que viajan con ofertas de viajes cerradas desde el origen (paquetes turísticos cerrados) adquiridos a través de las agencias de viajes. Estos paquetes de viaje no permiten al turista la elección del modo de viaje y por tanto no están recogidos en la encuesta de movilidad. Como la oferta de estos paquetes turísticos es cerrada, sería necesario un análisis en profundidad de tipo cualitativo de los Tour Operadores, Agencias de viajes e incluso de los Guías de la ciudad que generan esta oferta. El planteamiento de esta línea de investigación respecto a la movilidad del turista de la ciudad de Toledo deberá continuar analizando si sería viable la opción de viaje intermodal para este tipo de oferta de viajes cerrados siendo Toledo el inicio o el final de la ruta planteada como opción de viaje.

\section{REFERENCIAS BIBLIOGRÁFICAS}

Cokasova, A. (2003). Modelling of air-rail intermodality from passenger perspective at major European airports. (Unpublished doctoral dissertation). University of Zilina: Eurocontrol Experimental Centre.

Comisión Europea (2001). La política europea de transportes de cara al 2010: la hora de la verdad, Libro Blanco (COM, 2001, 370 final). Recuperado de http://eurlex.europa.eu/LexUriServ/LexUriServ.do?uri=CELEX:52001DC0370:ES:HTML

Comisión Europea (2004a). Towards Passenger Intermodality in the EU. - Report 1: Analysis of the Key Issues for Passengers Intermodality (Final Version, DG Energy and Transport). Recuperado de http://www.ils-forschung.de/down/towards-pass-1.pdf

Comisión Europea (2004b). Towards Passenger Intermodality in the EU.-Report 2: Analysis of the National Inventories for Passengers Intermodality (Final Version, DG Energy and Transport). Recuperado de http://www.ils-forschung.de/down/towards-pass-2.pdf 
Comisión Europea (2009). Un futuro sostenible para los transportes: hacia un sistema integrado, tecnológico y de fácil uso (COM, 2009, 279 Final). Recuperado de http://eurlex.europa.eu/LexUriServ/LexUriServ.do?uri=COM:2009:0279:FIN:ES:PDF

Comisión Europea (2011). Hoja de ruta hacia un espacio único europeo de transporte: por una política de transportes competitiva y sostenible. Libro Blanco de la Comisión Europea (COM 144 final). Recuperado de http://eurlex.europa.eu/LexUriServ/LexUriServ.do?uri=COM:2011:0144:FIN:ES:PDF

Eurocontrol (2005a). Potential Airport Intermodality Development, CARE II MODAIR, Measure and development of interMODality at AlRport. Recuperado de http://www.eurocontrol.int/care-innov/gallery/content/public/docs/projects20052006/modair/WP1\%201.1.pdf

Eurocontrol (2005b). Airport Intermodality Indicators", CARE II: MODAIR Measure and development of interMODality at AIRport, M3 SYSTEMS, ANA, ENAC-AEEL. Recuperado de http://www.eurocontrol.int/care-innov/gallery/content/public/docs/projects20052006/modair/WP2 1.1.doc

Martínez, T., y Muro, A.I. (2011). Caso de Estudio3: Corredor Toledo-Madrid, Tercer Informe de Seguimiento (Proyecto AEROAVE: La integración del transporte aéreo con las redes ferroviarias de larga distancia). Recuperado de http://www.aeroave.es/documentos.asp\#

Muro, A. I. (2012). Intermodalidad entre los modos aéreo y ferroviario: Aplicación al corredor Madrid-Toledo (Tesis Doctoral). Universidad de Castilla-La Mancha.

Nombela, G. (2011). La intermodalidad AEROAVE: Recomendaciones y propuestas para España (Proyecto AEROAVE, Documento no. 4, Septiembre). Recuperado de http://www.aeroave.es/documentos.asp\#

Nombela, G., Hernández, A., Ladouse, S., Herranz, R., y Sánchez, V. (2010). Primer Informe de Seguimiento Proyecto AEROAVE. (Proyecto AEROAVE, Documento no 1, Marzo). Recuperado de http://www.aeroave.es/documentos.asp\#

Pérez, I.R. (2014). Introducción a la Competencia en el proceso de liberalización del transporte ferroviario de viajeros en España. Un caso de estudio (Tesis Doctoral). Universidad de Castilla-La Mancha.

Para citar este artículo: Muro, A.I. y Jiménez, I.R. (2016). La intermodalidad aéreo-ferroviaria del turismo en la ciudad de Toledo. Investigaciones Turísticas, (12), 121-141. http://dx.doi.org/10.14198/INTURI2016.12.06 